$\therefore$.

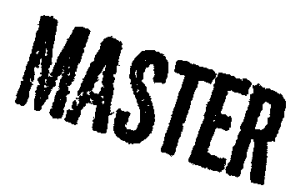

\title{
SPECIFICATION \\ REQUIREMENTS SUMMARY \\ FOR THE \\ BRAYTON ISOTOPE POWER SYSTEM (BIPS) \\ GROUND DEMONSTRATION SYSTEM (GDS)
}

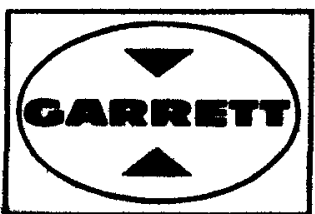




\section{DISCLAIMER}

This report was prepared as an account of work sponsored by an agency of the United States Government. Neither the United States Government nor any agency Thereof, nor any of their employees, makes any warranty, express or implied, or assumes any legal liability or responsibility for the accuracy, completeness, or usefulness of any information, apparatus, product, or process disclosed, or represents that its use would not infringe privately owned rights. Reference herein to any specific commercial product, process, or service by trade name, trademark, manufacturer, or otherwise does not necessarily constitute or imply its endorsement, recommendation, or favoring by the United States Government or any agency thereof. The views and opinions of authors expressed herein do not necessarily state or reflect those of the United States Government or any agency thereof. 


\section{DISCLAIMER}

Portions of this document may be illegible in electronic image products. Images are produced from the best available original document. 
Th s book was prepared as an account of work sponsored by an agency of the United States Government

Ne the the United States Government nor any agency thereof nor any of their employees makes any completeness or usefulness of any information apparatus product of process disclosed or
represents that is use commerce al product process or service by trade name trademark manufacturer or otherwise does
come ce not necessar ty constitute or imply its endorsement recommendation or favoring by the United
States Government or any agency thereof The $v$ dews and op noons of authors expressed herein do not necessar I $v$ state or reflect those of the Un ted States Government or any agency thereof

SPECIFICATION

REQUIREMENTS SUMMARY

FOR THE

DRAYTON ISOTOPE POWER SYSTEM (RIPS)

GROUND DEMONSTRATION SYSTEM (GDS)

$76-311737$

February 10, 1976

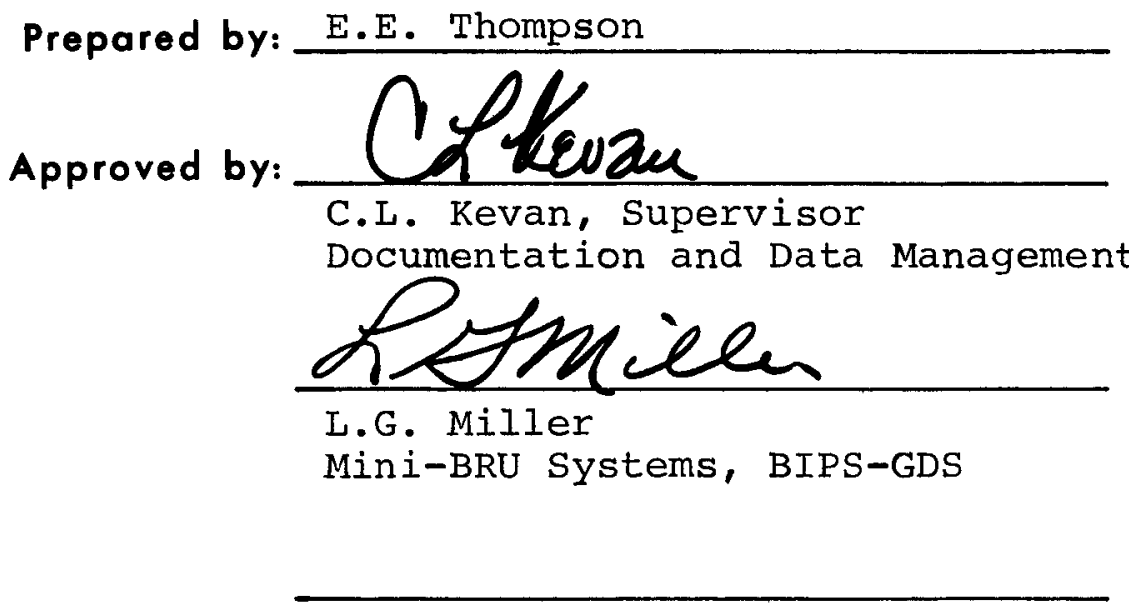

AIRESEARCH MANUFACTURING COMPANY OF ARIZONA

A DIVISION OF THE GARRETT CORPORATION

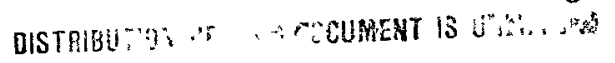




\section{SPECIFICATION \\ REQUIREMENTS SUMMARY \\ FOR THE \\ BRAYTON ISOTOPE POWER SYSTEM (BIPS) \\ GROUND DEMONSTRATION SYSTEM (GDS)}

\section{INTRODUCTION}

This document provides a summary of specification and procedure requirements for the ERDA Phase I Brayton Isotope Power system (BIPS) Program. Phase I covers design, fabrication, and test of the BIPS Ground Demonstration System (GDS) by AiResearch, Phoenix, under Contract No. $E(04-3)-1123$.

\subsection{Purpose}

The primary purpose of this document is to provide a summary of required program specifications and procedures. Also included are document definitions, descriptions, and formats, and a listing of commonly used abbreviations. This document is intended to be used as a guide in document preparation and control. The specification and procedure formats included herein reflect the applicable format requirements of MIL-STD-490. 


\section{DOCUMENT REQUIREMENT MATRIX/SUMMARY}

Overall document requirements for the BIPS GDS program have been assessed and are summarized in Table I. As noted in Table I, each component/subassembly and facility/equipment requirement has been itemized with a preliminary summary of the type of document required. It should be noted that in some instances a general procedure or specification will be prepared as the basic document, with appendices provided to cover unique requirements of specific hardware.

As an adjunct to Table I, an additional table (Table II) has been prepared to present a potential list of required documents by category, with a basic document number pre-assigned. A column is also provided that establishes estimated document preparation completion dates. 
3. DOCUMENT DEFINITION AND FORMAT

Documents required for the BIPS GDS program can essentially be divided into three major categories:

A. Specifications

1. Statement of Work (SOW)

2. Design Specifications (SC)

3. Procurement Specifications (PSC)

4. Material Specifications (EMS)

5. Equipment/Facility Specifications

6. Process Specifications

7. Fabrication Specifications

B. Procedures/Plans

1. Test Plans

2. Test Procedures

(a) Development Test Instructions (DTI)

(b) Formal Test Procedures

(c) Acceptance Test Procedures (ATP)

3. Inspection Procedures

4. Cleaning Procedures

5. Storage and Handling Procedures

6. Assembly Procedures (EAI)

C. Reports

1. Test Reports

(a) Development (DTR)

(b) Formal Test Repoirts

These major categories are discussed in detail in the following paragraphs. 


\subsection{Specifications}

Various types of specifications are required to define specific requirements related to a particular program. Specifications always define requirements. These are defined, in general, with basic formats as follows:

A. Statement of Work - defines the scope of work to be accomplished by a contractor or subcontractor during fulfillment of a particular contract. A sow is normally prepared when the work scope is of sufficient magnitude to involve all types of personnel, facilities, services, materials, technical support, including design, fabrication, testing, scheduling, and reporting. Actual deliverable hardware may or may not be involved.

FORMAT

1. INTRODUCTION

1.1 Purpose

1.2 Scope

2. APPLICABLE DOCUMENTS

3. SCOPE OF WORK

3.1 Task Definition

4. TECHNICAL REQUIREMENTS

5. GENERAL REQUIREMENTS

6. DELIVERY REQUIREMENTS

7. NOTES AND DEFINITIONS

B. Design Specification - defines detailed technical elements associated with the design of components, equipment, subsystems, and systems. 
FORMAT

1. INTRODUCTION

1.1 Purpose

1.2 Scope

2. APPLICABLE DOCUMENTS

3. DESIGN REQUIREMENTS

3.1 General Requirements

3.2 Functional Requirements

3.3 Design and Construction

3.4 Environmental

3.5 Safety/Reliability/Maintainability

4. QUALITY ASSURANCE PROVISIONS

5. PREPARATION FOR DELIVERY

6. NOTES AND DEFINITIONS ATTACHMENTS/APPENDICIES

C. Procurement Specification - a PSC is referenced on a Source or Specification Control Drawing and is an extension thereof, and defines technical requirements for subcontractors to design, fabricate, test, and deliver detail equipment, data, documentation, and services.

\section{FORMAT}

1. INTRODUCTION

1.1 Purpose

1.2 Intended Use

2. APPLICABLE DOCUMENTS

3. REQUIREMENTS

4. QUALITY ASSURANCE PROVISIONS

5. PREPARATION FOR DELIVERY

6. NOTES AND DEFINITIONS ATTACHMENTS/APPENDICIES 
D. Fabrication Specification - defines criteria for materials, processes, quality, and testing requirements for components, subassemblies, and assemblies designed by AiResearch or a subcontractor and manufactured by another subcontractor.

1. INTRODUCTION

1.1 Purpose

1.2 Intended Use

2. APPLICABLE DOCUMENTS

3. REQUIREMENTS

4. QUALITY ASSURANCE PROVISIONS

5. PREPARATION FOR DELIVERY

6. NOTES AND DEFINITIONS

E. Material Specifications - define requirements for materials including chemical composition, mechanical properties, process control, inspection, identification and packaging. Material specifications are prepared as required for AiResearch and subcontractor usage.

FORMAT

1. APPLICATION

2. APPLICABLE DOCUMENTS

3. TECHNICAL REQUIREMENT

3.1 Material

3.2 Chemical Composition

3.3 Mechanical Properties

3.4 Hardness

3.5 Grain Size 
3. 6 Ductility

4. PROCESS CONTROL

5. INSPECTION

6. IDENTIFICATION AND PACKAGING

7. APPROVAL OR PROCUREMENT

8. REPORTS

9. QUALITY CONTROL

F. Process Specification - defines requirements for material specifications wherein processes are defined such as process materials, plating, heat treating, manufacturing operations, inspection, and handling criteria. Process specifications are prepared for AiResearch and subcontractor usage.

FORMAT

1. INTRODUCTION

2. APPLICABLE DOCUMENTS

3. SPECIAL EQUIPMENT

4. MATERIALS

5. MANUFACTURING OPERATIONS

5.1 Cleaning

5.2 Plating

5.3 Preparation of Materials

5.4 Brazing and Heat Treating

6. INSPECTION

7. QUALITY CONTROL

G. Equipment/Facility Specification - defines distinct requirements for equipment or facilities, such as technical provisions, general requirements, acceptance, inspection, and checkout. 
AIRESEARCH MANUFACTURING CDMPANY DF ARIZONA

A DIVISION OF THE GARAETT CORPOAATION

PHOENIX, ARIZLNA

FORMAT - varies according to complexity of equipment or facility; however, a typical facility

format would include:

1. SCOPE OF WORK

2. DRAWINGS

3. SHOP DRAWINGS

4. SPECIFICATIONS

5. CHANGES

6. PERMITS AND REGULATIONS

7. MATERIAI AND EQUIPMENT

8. PROGRESS CHARTS/REPORTING

9. SUBCONTRACTING

10. WORK RESPONSIBILITIES

11. FACILITY REQUIREMENTS

12. INSPECTION

13. OPERATIONAL ACCEPTANCE TESTS

\subsection{Procedures/Plans}

Various types of procedures and plans are prepared to define methods of accomplishing a specific task. Procedures and plans always define the method of accomplishing a given task.

Specific types of procedures are defined and generally structured as follows:

A. Test Plan - defines the overall scope of test requirements, including development and demonstration testing. Included are administrative, quality assurance, inspection, test witnessing, general test setup schematics, and data requirements. 


\section{FORMAT}

1. INTRODUCTION

1.1 Purpose

1.2 Scope

2. TEST REQUIREMENTS

2.1 General Requirements

2.2 Test Procedures

2.3 Format

2.4 Pre-Test Inspection

2.5 Administrative Requirements

2.5.1 Test Witnessing

2.5 .2 Test Log

2.5.3 Test Data

2.5.4 Photographs

2.5.5 Test Reports

3. FACILITIES AND SERVICES

3.1 Test Facilities

3.1 .1 Test setup

3.1.2 Instrumentation

3.1.3 Instrumentation Accuracies

3.2 Services

4. TEST PROCEDURE

4.1 Test Conditions

4.2 Test Installation

4.3 Checkout

4.4 Malfunctions and Failures

4.5 Data Check

4.6 Post-Test Inspection

5. TEST PROGRAM

6. TEST SCHEDULE 
B. Test Procedures

1. Development Test Instruction (DTI) - provides detailed test instructions for use during development tests, and is an informal procedure prepared for AiResearch internal use only.

FORMAT

1. TEST ITEM AND PART NUMBER

2. TEST TITLE

3. FACILITIES AND SPECIAL TEST EQUIPMENT

4. TEST OBJECTIVE

4.1 Test Procedure

4.2 Data Required

2. Formal Test Procedure - provides detailed formal test instructions for conducting tests required by the procuring activity, or design specification. This type of procedure is normally provided to the procuring activity (customer).

FORMAT

1.1 Purpose

1.2 Scope

1.3 Administrative requirements

2. ACCEPT - REJECT CRITERIA

3. TEST

3.1 Special Instructions

3.2 Test Setup

3.3 Test Procedure 


\subsection{Data Requirements \\ 3. 5 Post-Test Inspection \\ Attachments}

Test Set-up Schematics

Data sheets

C. Inspection Procedure - defines detail inspection criteria for a component, subsystem, system, or as required by a detailed formal test procedure.

FORMAT

1. INTRODUCTION

1.1 Purpose

1.2 Scope

1.3 Applicability

2. GENERAL REQUIREMENTS

2.1 Inspection Criteria

2.2 Equipment, Processes, and Supplies

2.3 Critical Cards

2.4 Inspection Records

3. INSPECTION REQUIREMENTS

3.1 Parts, Components, and Assemblies

3.2 Inspection After Test

4. ADDITIONAL REQUIREMENTS

4.1 Witnessing

4.2 Parts Discrepancies

5. DEFINITIONS AND NOTES

D. Cleaning Procedure - defines detail cleaning requirements for a component, subsystem, system, as required by a specific application. 
FORMAT

1. SCOPE

2. APPLICABLE DOCUMENTS

3. CLEANING REQUIREMENTS

4. MATERIAL CONTROL

5. CLEAN ROOM CONTROLS

5.1 Cleaning Methods

5.2 Cleanliness Control

5.3 Contamination Limits

5.4 Test Media and Materials

5.5 Test Component Handling

5.6 Test Equipment Handling

5.7 Packaging

6. QUALITY ASSURANCE PROVISIONS

7. NOTES AND DEFINITIONS

E. Storage and Handling Procedure - defines the criteria for handling and storage of components, subsystems, systems, and test equipment wherein specific methods are required due to cleanliness, contamination, radioactivity, fragile materials, etc.

FORMAT

1. SCOPE

2. APPLICABLE DOCUMENTS

3. GENERAL REQUIREMENTS

4. SPECIFIC REQUIREMENTS

4.1 Handling Methods

4.2 Storage Methods

4.3 Recordkeeping

4.4 Periodic Inspection

5. PRESERVATION AND DEPRESERVATION

6. NOTES AND DEFINITIONS 


\section{F. Engineering Assembly Instruction (EAI) - provides}

Engineering instruction for assembly and test of modules, components, or subassemblies.

\section{FORMAT}

1. INTRODUCTION

1.1 Purpose

2. INSTRUMENTATION AND EQUIPMENT

3. PROCEDURE

\subsection{Test Reports}

Test reports are prepared to summarize and document test results on development production hardware. Test reports always describe results.

A. Development Test Report (DTR) - is prepared to summarize test results on development hardware only. Development test reports include data, conclusions, recommendations, recording traces, photographs, and other test data as applicable. DTR's are used for AiResearch internal purposes to establish or verify final design or performance criteria.

FORMAT

1. TEST ITEM AND PART NUMBER

2. TEST TITLE

3. TEST OBJECTIVE

4. TEST RESULTS

4.1 Summary

4.1.1 Test Goals

4.1.2 Test Results

4.1.3 Data.Analysis and Validity 
5. CONCLUSIONS AND RECOMMENDATIONS

Attachments

Schematics

Recording Traces

Photographs

Curves

Data Sheets

B. Formal Test Report - is prepared to summarize and document test results obtained as a result of testing conducted to a formal procedure. Reports are normally prepared to a format consistent with contract or procuring activity requirements. Reports are usually submitted to a customer to verify design and performance of an end item of hardware, i.e., component, subsystem, or system.

\section{FORMAT}

1. INTRODUCTION AND SUMMARY

2. DESCRIPTION OF TEST ARTICLE

2.1 Test History

2.2 Test Configuration

3. DESCRIPTION OF TEST

4. INSPECTION RESULTS

5. DISCUSSION OF TEST RESULTS

6. CONCLUSIONS AND RECOMMENDATIONS

Appendices

Photographs

Test Log and Recordings

Component Verification Test 
247-15

AIRESEARCH MANUFACTURING COMPANY OF ARIZLNA

A DIVISION OF THE GARAETT COAPORATION

PHIENIX, ARIZONA

4. ABBREVIATIONS AND ACRONYMS

Table III provides a general listing of abbreviations and acronyms as related to the BIPS GDS program. 
TABLE

SPECIFTCATION/PROCEDURE REQUIREMENTSS SUMMARY

\begin{tabular}{|c|c|c|c|c|c|c|c|c|c|c|c|c|c|c|c|c|c|c|}
\hline \multicolumn{19}{|c|}{ 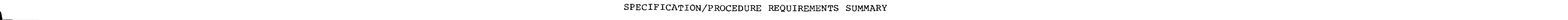 } \\
\hline 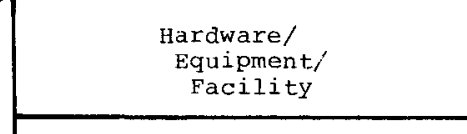 & $\begin{array}{c}\text { Pesponsibility } \\
\text { Supplier }\end{array}$ & 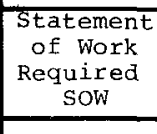 & $\begin{array}{c}\text { Procurement } \\
\text { specs. } \\
\text { pSC }\end{array}$ & $\begin{array}{c}\text { Equip/ } \\
\text { Facility } \\
\text { spec. } \\
\text { spec }\end{array}$ & $\begin{array}{l}\text { Fab. } \\
\text { speec } \\
\text { spen }\end{array}$ & $\begin{array}{c}\text { System/ } \\
\text { subsystem } \\
\text { spece. }\end{array}$ & 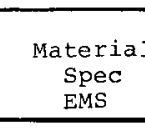 & 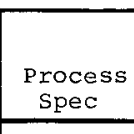 & Inspection & cleaning & \begin{tabular}{|l}
$\begin{array}{r}\text { Storage } \\
\text { and } \\
\text { Handing }\end{array}$ \\
\end{tabular} & EAI's & 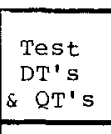 & $\begin{array}{c}\text { Test } \\
\text { Report } \\
\end{array}$ & $\begin{array}{l}\text { Test } \\
\text { Plan } \\
\end{array}$ & Note & $\begin{array}{l}\text { Comnenténent } \\
\text { Regui rement }\end{array}$ & $\begin{array}{l}\text { Responsible } \\
\text { Engineer }\end{array}$ \\
\hline 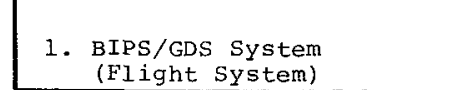 & Air phx & -- & -- & -- & -- & $\mathrm{x}$ & -- & -- & $x^{1}$ & (1) & $x$ & $x$ (1) & 急 & $\times(1)$ & $x(1)$ & (1) & 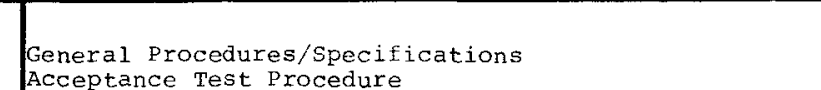 & E. Ro11 \\
\hline 2. Mini--BRU & GFE & -- & - & -- & - & $\overline{--}$ & -- & -- & $x^{2}$ & $x^{2}(2)$ & $x^{(2)}$ & $-\bar{x}$ & $x^{(2)}$ & $\times(2)$ & $x^{(2)}$ & 2 & $\begin{array}{l}\text { Includuded in General proceedure (1), or } \\
\text { as procodure Appendice. }\end{array}$ & F. Dobler \\
\hline 3. Recuperator & ${ }_{G F E}$ & -- & $\ldots$ & $\ldots$ & $\ldots$ & $\ldots$ & $\ldots$ & $\ldots$ & $x^{2}(2)$ & $x^{2}(2)$ & $x^{2}(2)$ & & $x(2)$ & $\times(2)$ & $x \times$ & & & T. Ashe \\
\hline 4. $\mathrm{HSA} / \mathrm{EHS}$ & GFE & $\ldots$ & $\ldots$ & $\ldots$ & $\ldots$ & $\ldots$ & $\ldots$ & $\ldots$ & $\times 2$ & $\times(2)$ & $\times(2)$ & $x^{(2)}$ & $x$ (2) & $x^{(2)}$ & $x^{(2)}$ & - & & T. Ashe/P. Post \\
\hline 5. Refractory Plenum & ${ }_{\text {GFE }}$ & 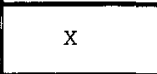 & -- & -- & -- & -- & -- & -- & $x^{(2)}$ & $x^{(2)}$ & $\times(2)$ & $x^{(2)}$ & $\mathrm{x}^{2}$ & $x^{2}$ & $\left.x^{2}\right)^{2}$ & (3) & 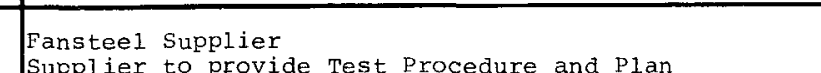 & F. Dobler \\
\hline 6. Heat Exchanger & $G \mathrm{E}$ & $\mathrm{x}$ & -- & -- & -- & (4) & -- & -- & $x^{2}(2)$ & $x^{2}(2)$ & $x^{2}(2)$ & -- & (3) & (3) & $\mathrm{x}^{(3)}$ & (4) & 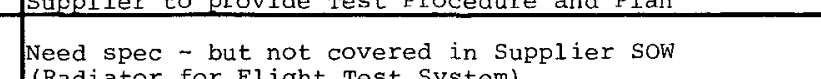 & T. Ashe \\
\hline 7. Multi-Foil Insulation & $\mathrm{TEECO}$ & & $\ldots$ & -- & & $\ldots$ & (4) & $\ldots$ & $\times(2)$ & $x^{2}(2)$ & $\times(2)$ & (4) & $x(2)$ & $x^{2}(2)$ & $x 3^{3}$ & & & T. Ashe \\
\hline $\begin{array}{ll}8 . \text { Bel 10ws } & \text { T }\end{array}$ & AiR LA & $\times(5)$ & -- & -- & $\times($ ) & -- & -- & $\overline{--}$ & $x^{2}$ & $x^{2}$ & $x^{2}$ & $x(7)$ & - & -- & $x^{(2)}$ & 8 & 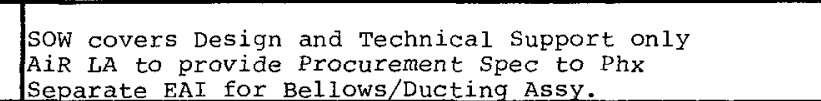 & T. Ashe \\
\hline 9. weld Joint & GE \& AiR Phx & $\ldots$ & & $\ldots$ & -- & -- & $x(8)$ & $x^{(8)}$ & $x^{2}(2)$ & $x^{2}(2)$ & $\times(2)$ & $x_{x}^{(7)}$ & -. & ב- & $x^{2}(2)$ & 8 & May be part of Source Control Drawing (SCD) & J. Had1ey \\
\hline 10. Ducting & Air phx & 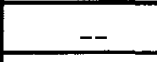 & $\times(8)$ & $\ldots$ & $\ldots$ & $\ldots$ & $x$ & $\ldots$ & $x^{2}(2)$ & $x^{2}(2)$ & $x(2)$ & $x(0)$ & $\ldots$ & -- & $x^{(2)}$ & & & J. Hadley \\
\hline 11. Assemb1y $\leftrightharpoons$ & Air phx & $\ldots$ & -- & $\ldots$ & $\ldots$ & $\ldots$ & & $\ldots$ & $\times(2)$ & $x^{(2)}$ & $\times(2)$ & $x(7)$ & $\ldots$ & $\ldots$ & ${ }_{x}^{(2)}$ & & & T. Ashe \\
\hline 12. Support structure & AiR Pbx & 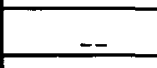 & & & & & 8 & & $-\frac{2}{2}$ & $-(2)$ & $x \times(2)$ & $x(2)$ & & & $\ldots$ & & & T. Ashe \\
\hline 13. Working Fluid & Ais Phx & - & & & - & & & & (2) & $\times 2$ & $\times 2$ & & & & - & & & J. Haaley \\
\hline 14. control system & AiR Phx & - & $x(9)$ & -- & - & $\times(1)$ & $x$ (11) & -- & $x^{2}$ & $\times 2$ & $x^{2}$ & $x$ (12) & $\times(13$ & $\times(13)$ & $\overline{--}$ & 8 & 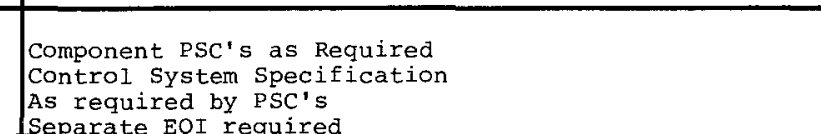 & P. Post \\
\hline \multirow{2}{*}{\begin{tabular}{|l|l} 
15. Test Materials \\
Joints \\
Insulation \\
Material samples \\
\end{tabular}} & & -- & 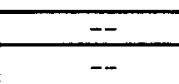 & $\underline{--}$ & $\cdots$ & & -2 & $\ldots$ & - & $=-$ & -- & -- & 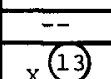 & 8 & $\ldots$ & 3 & $7^{\text {PT's }^{\prime} \text { and DTR ' }}$ & \multirow[b]{2}{*}{ T. Ashe } \\
\hline & & -- & -- & - & - & -- & $\frac{x}{8}$ & $\frac{x}{x}$ & -- & 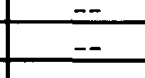 & $\ldots$ & $=-$ & $\frac{\frac{x+3}{x(3)}}{\frac{3}{3}}$ & $\frac{8(3)}{\frac{8}{83}}$ & 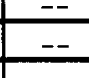 & 二 & & \\
\hline 16. Mockup & AiR phx & -- & $-\overline{-}^{-}$ & -- & -- & -- & $x$ & $x_{-1}$ & $\ldots$ & $\ldots$ & $\ldots$ & $\ldots$ & 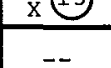 & $x-$ & $\cdots$ & & & T. Ashe \\
\hline \multicolumn{18}{|l|}{ FACILITTES/EQUTPMENT } & \multirow{4}{*}{ E. Ro11 } \\
\hline \multirow{3}{*}{$\begin{array}{l}\text { 1. Clean Facility } \\
\text { A. Clean Assy Area } \\
\text { Fi1ters Ars } \\
\text { Degreaser } \\
\text { Bake Oven }\end{array}$} & \multirow{12}{*}{ 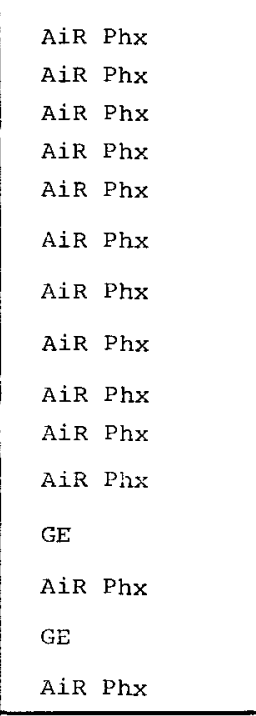 } & $\because-$ & $\ldots$ & $\frac{x(1)}{x(14)}$ & $\ldots$ & $\cdots$ & & & 一 $\times \frac{13}{15}$ & 一下年 & & & 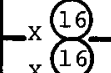 & & $\cdots$ & \multirow{2}{*}{ 急 } & \multirow{2}{*}{ 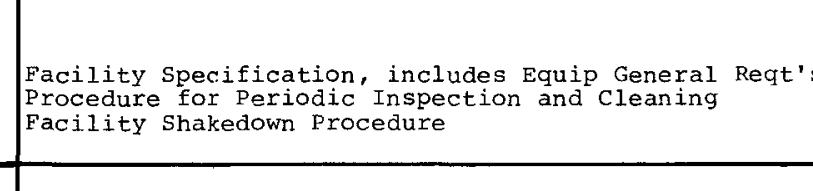 } & \\
\hline & & - & - & & - & $\cdots$ & $\ldots$ & $\ldots$ & & & $\ldots$ & $\ldots$ & & -- & $\cdots$ & & & \\
\hline & & $-\bar{c}$ & -- & -- & - & 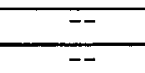 & -- & $=$ & -- & $=$ & -- & $\ldots$ & - & -- & $=$ & & - & \\
\hline B. Vac. Tank \& Pumps & & -- & $x$ (11) & $\overline{---}$ & $\ldots$ & -- & $\ldots$ & - & (15) & (15) & $\ldots$ & -- & (18) & -- & $\ldots$ & ه & Vyacuun rank spec to incl luae pumps & T. Ashe \\
\hline c. vac. Dry во.: & & $-\overline{-}$ & 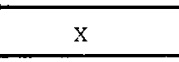 & -- & -- & $-\overline{-}$ & $\ldots$ & & $\times(15)$ & 8 (15) & - & $\ldots$ & (1) & $\ldots$ & $\ldots$ & 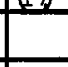 & & T. Ashe \\
\hline D. Facility Power & & -- & $\overline{--}$ & (14) & -- & -- & -- & -- & $\ldots$ & -- & -- & $\ldots$ & -- & $\overline{--}$ & 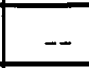 & (14) & Fart of Facility Spec & P. Post \\
\hline $\begin{array}{l}\text { E. Mechanical } \\
\text { Interface Equis, }\end{array}$ & & $\ldots$ & & (14) & $\ldots$ & $\ldots$ & $\bar{c}$ & 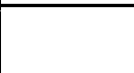 & 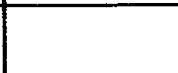 & & & & & . & $\ldots$ & (14) & ity spec & T. Ashe \\
\hline F. Hround Test Consic1e 18 & & $\overline{--}$ & $x(9)$ & -- & $\overline{--}$ & $\overline{--}$ & $\ldots$ & $\ldots$ & & (15) & & $\times 18$ & (16) & & $\ldots$ & (68) & Assembly (EAI) Instructions (if recuired) & T. Ashe \\
\hline G. Power Supply Corsole & & $x(2)$ & -- & -- & - & - & & & $x^{2}(2)$ & $\times(2)$ & $\times(2)$ & $\ldots$ & $\times(3$ & $\times(3)$ & $\times(3)$ & & Covared hy G5 S50w $75-3113750$ & p. Post \\
\hline H. Data Acquisition & & & $\ldots$ & $x$ (114) & -- & $\ldots$ & $\ldots$ & & & & & $\ldots$ & (11) & & & (14) & Include in Facility Spec (4) & B. MinshaI1 \\
\hline I. Gas Management system & & $x$ (19) & -- & -- & -- & -- & $\ldots$ & $\ldots$ & $x^{2}(2)$ & $x^{2}(2)$ & $x^{2}(2)$ & - & $\times(3)$ & $\times(3)$ & $x^{(3)}$ & & & r. Ashe \\
\hline J. Test Equipment & & -- & -- & & -- & $\overline{--}$ & $\ldots$ & $\ldots-$ & $\frac{x}{x(2)}$ & $x^{2}$ & $x^{2}(2)$ & $\ldots$ & $\frac{1}{x(10)}$ & -- & -- & & $\int_{\text {Minima1 }}$ Proced & T. Ashe \\
\hline 2. Contro1s Test Rig & AiR Phx & -- & $\overline{--}$ & $x(20)$ & -- & $\overline{--}$ & $\ldots$ & $\ldots$ & $x^{2}(2)$ & ne 40 & -- & $\ldots$ & & 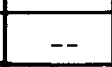 & $\ldots$ & (29) & Equipment specifications as required & P. Post \\
\hline 3. Facility Checkout & Air Phx & -- & $\overline{--}$ & -- & -- & -- & -- & -- & - & -- & -- & $\overline{--}$ & $x^{(16)}$ & -- & $-\overline{-}$ & & Part of Facility Shakedown Procedure & G. suchnan \\
\hline
\end{tabular}


TABLE II

Page 1 of $?$

SPECIFICATION/PROCEDURE REOUIREMENTS

BY DOCUMENT CATEGORY

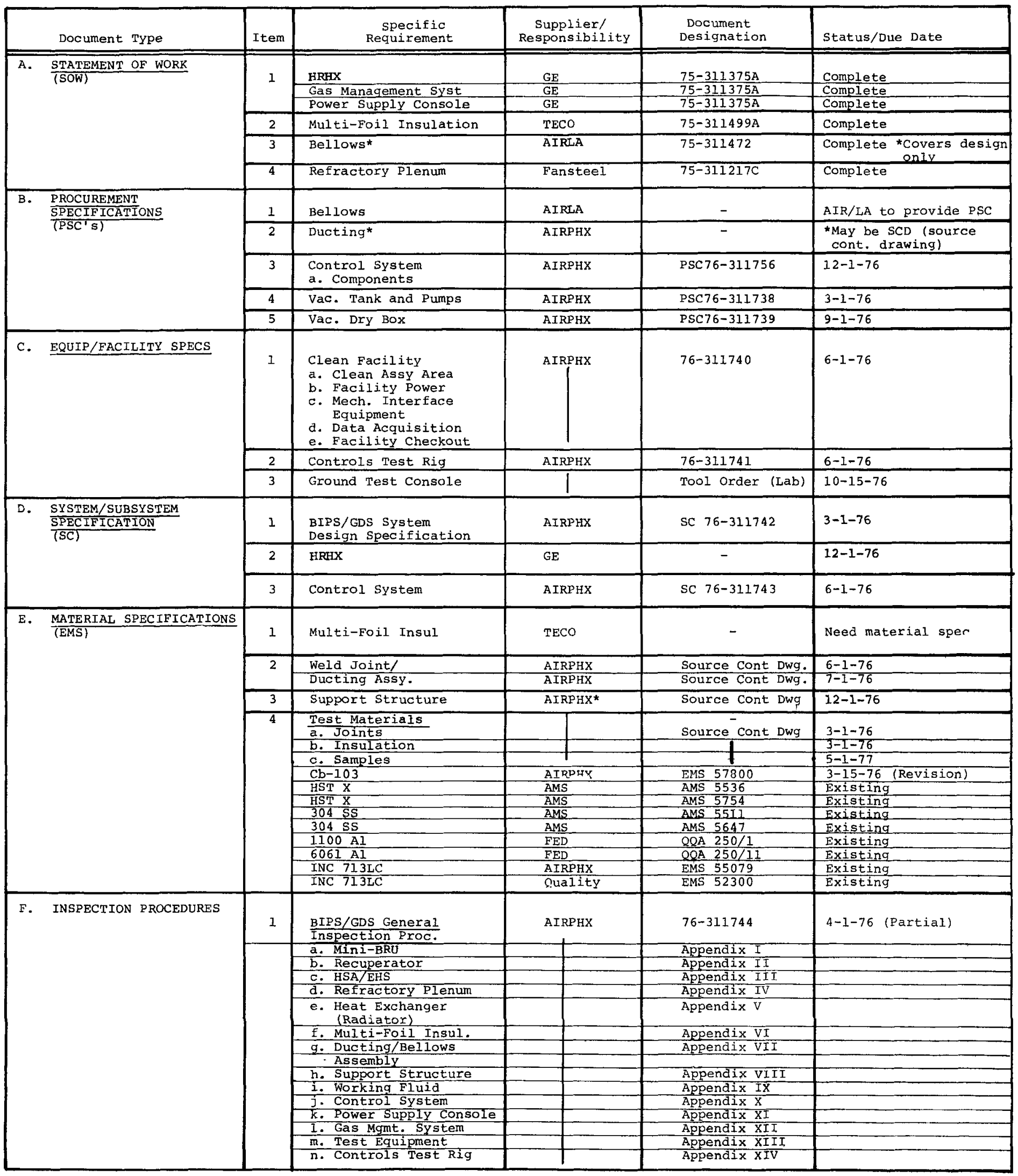




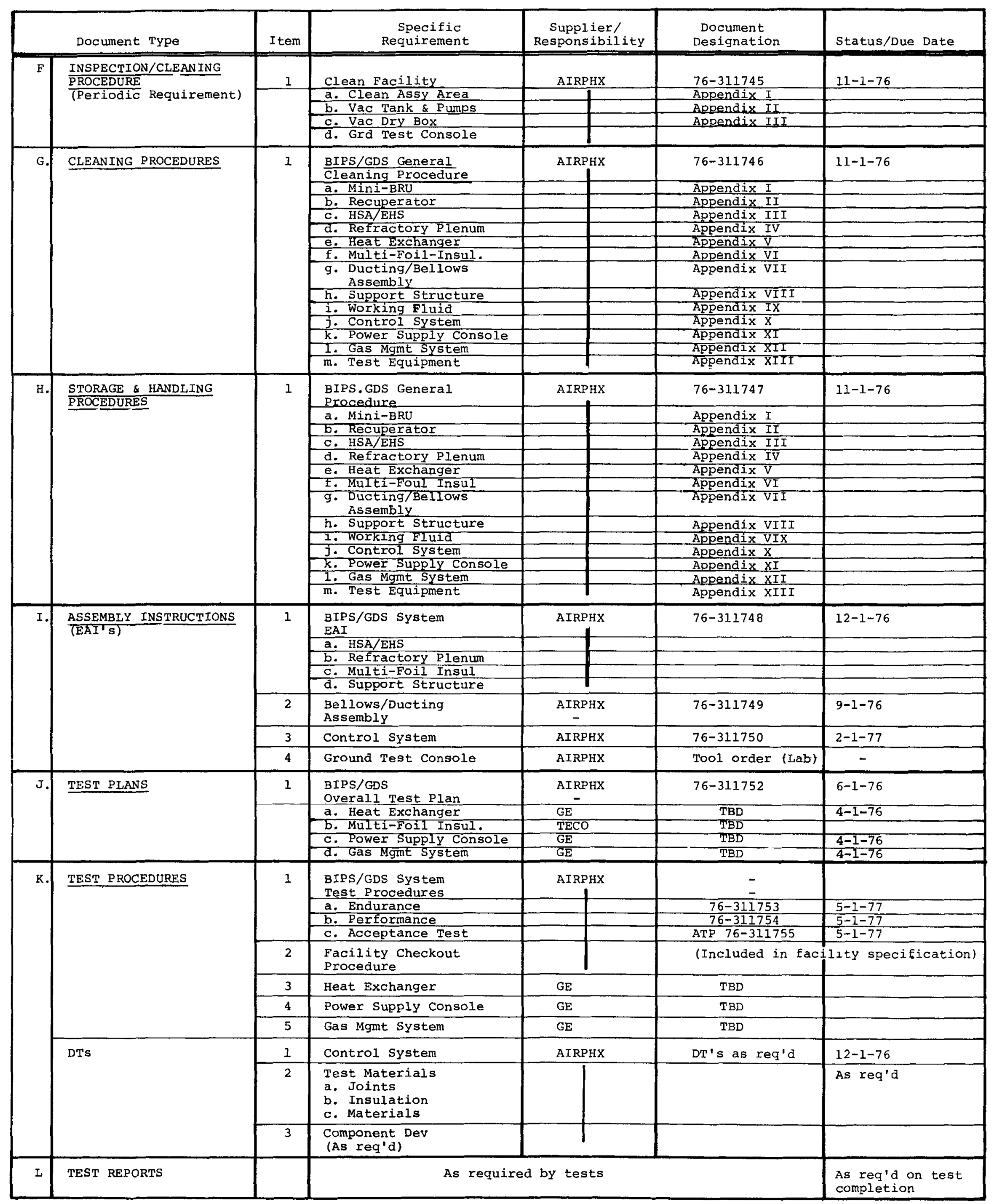


(A)

CFM

c.g. or $\mathrm{CG}$

$\mathrm{CO} 2$

CRT

Cu. Ft.

CSI

DAS Data Acquisition System

db Decıbel

DC Direct Current

DCS Demonstrator Cooling System

Delta-V or $\Delta V$ Velocity Cnange in Feet per Second

DOD

DOD STS

EAI

EAFB

EPFS

ECLS

ECS

EHS-D

EHS-S

ELS

EMS

$E O M$

ESD

ET

EPS

ERDA

EVA

Auxiliary Battery System

Auxiliary Cooling System (for HSA)

Control System

AlResearch, Phoenlx

de Modulation

Alrborne Support Equipment

Control Subsystem

Battelle Columbus Laboratory

Brayton Isotope Power System

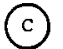

Centigrade Degrees

Cane Caveral Alr Force Station

Cathode Ray Tube

ubic Feet

Department of Defense

(E)

Englneering Assembly Instructions

Edwards Alr Force Base

Emergency Cooling System

Electrical Heat Source - Dynamic

Electrical Heat Source - Statıc

Eastern Launch Site

End of Mission

Electronic System Division

(of Alr Force System Command)

External Expendable Tank

Electrıcal Power System

Extravehicular Activity

\section{(E)}

Fahrenhelt Degrees Fuel Impact Body

Frequency Modulation

Fallure Mode Analysis

Fallure Modes and Effects Analysis

Federa $\&$ procurement Regulation

Feet per second

Flight System

Fuel Sphere Assembly

Final Safety Analysis Report

Flight Support Equipment

Follow-up Output

Feet, Foot

Food, Water and Waste Management (now ERDA)

1Research, Los Angeles Heat Transfer Systems

Atmospheric Revitalization Subsystem

Abort-To-Orbit

Department of Defense Space Transportation system

Electrically Powered Filght System (PCS + EHS-D)

Environmental Control and L1 fe Support

Material Specification (AlResearch Term)

Energy Research and Development Administration

Flight Battery System Required for start in Orbit

Fallure Modes, Effects and Criticality Analysis
GDEHS

GDS

$G E$

GET

GFE

GMS

GMT

GN2

GNEC

GSE

GTC
Acceleration Due to Gravity

Graphite Dynamic Electric Heat Source (GE Term Eor EHS-D) (Obsolete Term)

Ground Demonstration System - PCS + EHS-S

General Electric, Valley Forge General Electric Heat Source (GE term for EHS-S) (Obsolete Term)

round Elapsed Time

Covernment Furnished Equipment

Gas Management System

Greenwich Mean Time

Gaseous Nitrogen

Guldance, Navigation and Control

Ground Support Equipment

Ground Test Console (a STE/GSE 1 tem)

\section{(H)}

Heat Source (Same as and replaced by IHS)

Heat Source Assembly (Does not include IHS or HSS Does include HSHX, structure, insulation, ACS and ECS.)

Heat Source Heat Exchanger

Heat Source Insulation System

Heat Source Simulator (Same as and replaced by EHS) Hertz (Cycles)

\section{(I)}

Isotope Brayton Flight System

Isotope Brayton Ground Demonstration System (GDS + IHS)

Isotope Handling Equipment

Isotope Heat Source (Isotope and Reentry Protection)

Inertial Measurement Unit

Inter $1 \mathrm{~m}$ upper Stage

Intra Vehicular Activity

\section{(J)}

Jet Propulsion Laboratory (Pasadena, Cal.) Johnson Space Center (Houston)

\section{(I)}

1,000

Keyboard

Code elements per second in $1,000^{\prime} \mathrm{s}$

Bits per Second in $1,000^{\prime} \mathrm{s}$

Kilogram

Kalometers

Kennedy Space Center

Kilowatt Hours

\section{(I)}

Los Alamos Scientific Laboratory

Launch Control Center

Lincoln Experimental Satellite (No. B \& 9)

pounds

Load Factor

Liquid Hydrogen

Lithium Hydroxide

L1quid Oxygen

Launch Processing system

(M)

Meters

Mini-Brayton Recuperator

400 Watt Minl-Brayton-Economlc Radiolsotope Thermodynamic Generator Design (Refer also to IBFS)

Mission Control Center

Multiplexer-Demultiplexer

Masn Engune Cut-off

Multi-Hunared Watt (AEC/GE IHS Technology)

Megahertz

Millimeters

Mean Mission Duration

Mission Operation System

Milliradians

Mission Specialist Station 
(N)

NA

Not Applicable

NASA IeRC National Aeronautical and Space Administration Lewis Research Center

NRA

N.MI or Nautical Mile Nuclear Research and Application

(0)

OBF Orbiter Processing system

OHA Operational Hazard Analysis

ORNL Oak Ridge National Laboratory

OMS Orbital Maneuvering Subsystem

(P)

PCL Power Conversion Loop (Use of PCS preferred)

PCM Pulse Code Modulation

PCR Payload Changeout Room

PCS I Power Conversion System (HSA + BRU + MBR + Heat

sink + Controls + Piping \& Structure

PCS II Power Conversion System (HSA + BRU + MBR + Radiator

PGHM Payload Ground Handling Mechanism

PHA Preliminary Hazard Analysis

PLC Parasitic Load Control System

P/L Payload

PLCC Payload Control Center

PLH Payload Handling

PLM Payload Management

POP Perpendicular to Orb2t Plane

PSAR Preliminary Safety Analysis Report

PSC Power supply Console (for RHS)

PSC Procurement Specification (A1Research Term)

PSE Peculiar Support Equipment

psi Pounds per Square Inch

psia Pounds per square Inch Absolute

PSS Payload Specialist Station

PSSR Preliminary System Safety Review

(Q)

$q$ or $Q \quad$ Aerodynamic Pressure

QA Quality Assurance

(R)

RCS Reaction Control System

RCS Radiator Cooling System

RF Radio Frequency

RM Remote Manipulator

RMS Remote Manipulator System

RSS Root Sum Square

RTLS Return to Launch Site

RTG Radiosotope Thermoelectric Generator

RTS Remote Tracking Station

(s)

SAMSO

SNS

SMAB

$S R M$

SSP

SSME

SSS

SSPP

SSV

STDN/TDRSS

$\operatorname{STE}$

STS

SURVASCATCOM Alr Force Survivable Communication Satellite

[now AFSAT (COM III)

Space and Missile System Organization

Space Nuclear Systems

Solld Motor Assembly Bulldup

Solid Rocket Booster

Solld Rocket Motor

Space Shuttle Program

Space Shuttle Main Englne

Space Shuttie System

System Safety Program Plan

Space Shuttle Vehicle

Space Trackıng and Data Network/Trackıng Data Relay Satellite System

Special Test Equipment
To Be Defined

Temperature of Cabin

Tracking and Data Relay Satelizte

Teledyne Energy Systems (formeriy Teledyne Isotopes

Turbine Inlet Temperature

Television

(U)

ULT Ultimate Loads

USAR Updated Safety Analysis Report

(v)

VAB

VAFB

VDC

$\mathrm{VHF}$

VRE

Vehicle Assembly Building

Vandenberg Alx Force Base

Volts Direct Current

Very H1gh Frequency

Voltage Regulator Exclter 